\title{
Lie Algebra Classification, Conservation Laws, and Invariant Solutions for a Generalization of the Levinson-Smith Equation
}

\author{
G. Loaiza $\mathbb{D}^{1},{ }^{1}$ Y. Acevedo $\left(\mathbb{D},{ }^{1}\right.$ O.M.L. Duque ${ }^{\mathbb{D}},{ }^{1}$ and Danilo A. García Hernández $\mathbb{D}^{2}$ \\ ${ }^{1}$ Universidad EAFIT, Medellín, Colombia \\ ${ }^{2}$ IMECC-UNICAMP, Campinas, Brazil
}

Correspondence should be addressed to Y. Acevedo; yaceved2@eafit.edu.co

Received 3 January 2021; Revised 2 April 2021; Accepted 22 April 2021; Published 7 May 2021

Academic Editor: Peiguang Wang

Copyright (c) 2021 G. Loaiza et al. This is an open access article distributed under the Creative Commons Attribution License, which permits unrestricted use, distribution, and reproduction in any medium, provided the original work is properly cited.

We obtain the optimal system's generating operators associated with a generalized Levinson-Smith equation; this one is related to the Liénard equation which is important for physical, mathematical, and engineering points of view. The underlying equation has applications in mechanics and nonlinear dynamics as well. This equation has been widely studied in the qualitative scheme. Here, we treat the equation by using the Lie group method, and we obtain certain operators; using those operators, we characterized all invariants solutions associated with the generalized equation of Levinson Smith considered in this paper. Finally, we classify the Lie algebra associated with the given equation.

\section{Introduction}

Lie group symmetry method is a powerful tool employed to study ODEs, PDEs, FPDEs, FODEs, and so on. This theory was introduced in the 19th century by Sophus Lie [1], following the idea of Galois theory in algebra. Lie group method applied to differential equations has received great interest among researchers in different fields of science such as mathematics, theoretical, and applied physics, due to the physical interpretations of the underlying equations that are studied. As a consequence, this method leads to construct, for example, conservation laws, using the well known Noether's theorem [2], even more applying Ibragimov's approach [3]. In the same way, it is possible to build similarity solutions which, in the traditional methods, are not possible.

Furthermore, this method contributes to establish schemes and the usefulness of some numerical methods; here, many packages are being built in different environments of computations, e.g., $[4,5]$. In general, taking into account the importance of the equations' study (such as ODEs, PDEs, and others), this method can be interesting to different researchers. A vast reference in Lie group method can be found in the literature, e.g., [6-9]. Recently, the Lie group method approach has been applied to solve and analyze different problems in many scientific fields, e.g., in [10], the authors applied the Lie symmetry method to investigate a fourth-order $1+2$ evolutionary partial differential equation which has been proposed for the image processing noise reduction. References in the latest progress in symmetry analysis can be found in [11-18] and therein.

In [19], Kamke proposes the following differential equation:

$$
y_{x x}-\frac{f_{y}(y)}{f(y)} y_{x}^{2}+g(x) y_{x}+h(x) f(y)=0,
$$

where $f, g, h$ are the arbitrary functions, for this equation presents the transformation $y(x)=\eta(\xi(x))$ which reduces this equation to a system of two first-order equations. Equation (1) can be written as

$$
y_{x x}+\phi\left(x, y, y_{x}\right) y_{x}=\gamma(x, y),
$$

where $\quad \phi\left(x, y, y_{x}\right)=-\left(\left(f_{y}(y) / f(y)\right) y_{x}+g(x)\right) \quad$ and $\gamma(x, y)=-h(x) f(y)$. This means that the coefficient of friction, i.e., $\phi$ is a function that depends on $x, y$, and $y_{x}$, and it will almost always be a nonlinear function, and on the other hand, function $\gamma$, which is known as function of 
disturbance, is also nonlinear. It is worth remembering that this type of equation (2) is known as generalizations of the Levinson-Smith equation. Also, equation (2), which is a particular case of the generalized Levinson-Simth equations, is related to the Liénard-type second-order nonlinear differential equation [20]. The underlying equations describe several phenomena in different areas such as electronics, biology, mechanics, seismology, chemistry, physics, and cosmology, for example, an important model in physical and biological sciences is the van der Pol equation, which describes a nonconservative oscillator with nonlinear damping. Levinson and Smith in [21] studied a general equation for relaxation oscillations.

In [8], the equation,

$$
y_{x x}=2 y_{x}^{2} y^{-1}+x^{-1} y_{x}+x^{-1} y^{2},
$$

is presented; note that equation (3) is a particular case of equation (1), with $f(y)=y^{2}, g(x)=-x^{-1}$ and $h(x)=-x^{-1}$; in [8], Cantwell states that equation (3) has a group of symmetries 5-dimensional; but it does not exhibit the development of said statement; they affirm the Lie group of symmetries of (3), using a ODEtools Maple package. In fact, the goal of this work is (i) to calculate the 5-dimensional Lie symmetry group in detail, (ii) to present the optimal system (optimal algebra) for (3), (iii) to make use of all elements of the optimal algebra to propose invariant solutions for (3), then (iv) to construct the Lagrangian with which we could determine the variational symmetries using Noether's theorem and thus to present conservation laws associated, and (iv) also to build some nontrivial conservation laws using Ibragimov's method, and finally (v) to classify the Lie algebra associated to (3), corresponding to the symmetry group.

\section{Continuous Group of Lie Symmetries}

In this section, we study the Lie symmetry group for (3). The main result of this section can be presented as follows.

Proposition 1. The Lie symmetry group for equation (3) is generated by the following vector fields:

$$
\begin{aligned}
& \Pi_{1}=-x \frac{\partial}{\partial x}+y \frac{\partial}{\partial y}, \\
& \Pi_{2}=y^{2} \frac{\partial}{\partial y}, \\
& \Pi_{3}=-x \frac{\partial}{\partial x}+x y^{2} \frac{\partial}{\partial y}, \\
& \Pi_{4}=x^{2} y^{2} \frac{\partial}{\partial y}, \\
& \Pi_{5}=x^{3} \frac{\partial}{\partial x}-2 x^{2} y+x^{3} y^{2} \frac{\partial}{\partial y} .
\end{aligned}
$$

Proof. A general form of the one-parameter Lie group admitted by (3) is given by

$$
\begin{aligned}
& x \longrightarrow x+\varepsilon \xi(x, y)+O\left(\varepsilon^{2}\right), \\
& y \longrightarrow y+\varepsilon \eta(x, y)+O\left(\varepsilon^{2}\right),
\end{aligned}
$$

where $\epsilon$ is the group parameter. The vector field associated with the group of transformations shown above can be written as $\Gamma=\xi(x, y)(\partial / \partial x)+\eta(x, y)(\partial / \partial y)$, where $\xi, \eta$ are differentiable functions in $\mathbb{R}^{2}$. Applying its second prolongation,

$$
\Gamma^{(2)}=\Gamma+\eta_{[x]} \frac{\partial}{\partial y_{x}}+\eta_{[x x]} \frac{\partial}{\partial y_{x x}},
$$

to equation (3), we must find the infinitesimals $\xi, \eta$, satisfying the symmetry condition,

$$
\begin{aligned}
\xi\left(x^{-2} y^{2}+y_{x} x^{-2}\right) & +\eta\left(2 y_{x}^{2} y^{-2}-2 x^{-1} y\right) \\
& +\eta_{[x]}\left(-4 y^{-1} y_{x}-x^{-1}\right)+\eta_{[x x]}=0,
\end{aligned}
$$

associated with (3). Here, $\eta_{[x]}, \eta_{[x x]}$ are the coefficients in $\Gamma^{(2)}$ given by

$$
\begin{aligned}
\eta_{[x]} & =D_{x}[\eta]-\left(D_{x}[\xi]\right) y_{x}=\eta_{x}+\left(\eta_{y}-\xi_{x}\right) y_{x}-\xi_{y} y_{x}^{2} \\
\eta_{[x x]} & =D_{x}\left[\eta_{[x]}\right]-\left(D_{x}[\xi]\right) y_{x x}, \\
& =\eta_{x x}+\left(2 \eta_{x y}-\xi_{x x}\right) y_{x}+\left(\eta_{y y}-2 \xi_{x y}\right) y_{x}^{2}-\xi_{y y} y_{x}^{3}+\left(\eta_{y}-2 \xi_{x}\right) y_{x x}-3 \xi_{y} y_{x} y_{x x} .
\end{aligned}
$$

Being $D_{x}$ as the total derivative operator, $D_{x}=\partial_{x}+y_{x} \partial_{y}+y_{x x} \partial_{y_{x}}+\cdots$. Replacing (8) into (7) and using (3), we obtain 


$$
\begin{aligned}
& \left(-\xi_{y y}-2 y^{-1} \xi_{y}\right) y_{x}^{3}+\left(\eta_{y y}-2 \xi_{x y}-2 y^{-1} \eta_{y}-2 \xi_{y} x^{-1}+2 y^{-2} \eta\right) y_{x}^{2} \\
& +\left(2 \eta_{x y}-\xi_{x x}-x^{-1} \xi_{x}+x^{-2} \xi-3 \xi_{y} x^{-1} y^{2}-4 y^{-1} \eta_{x}\right) y_{x} \\
& +\left(\eta_{x x}-x^{-1} \eta_{x}-2 x^{-1} y \eta+x^{-1} y^{2} \eta_{y}-2 x^{-1} y^{2} \xi_{x}+x^{-2} y^{2} \xi\right)=0 .
\end{aligned}
$$

From (9), canceling the coefficients of the monomial variables in derivatives $1, y_{x}^{3}, y_{x}^{2}$, and $y_{x}$, we obtain the determining equations for the symmetry group of (3). That is,

$$
\begin{array}{r}
y \xi_{y y}+2 \xi_{y}=0 \\
y^{2} x \eta_{y y}-2 y^{2} x \xi_{x y}-2 y x \eta_{y}-2 y^{2} \xi_{y}+2 x \eta=0 \\
2 x^{2} y \eta_{x y}-x^{2} y \xi_{x x}-x y \xi_{x}+y \xi-3 x y^{3} \xi_{y}-4 x^{2} \eta_{x}=0 \\
x^{2} \eta_{x x}-x \eta_{x}-2 x y \eta+x y^{2} \eta_{y}-2 x y^{2} \xi_{x}+y^{2} \xi=0
\end{array}
$$

Solving the system of equations (9a)-(9d) for $\xi$ and $\eta$, we get

$$
\begin{aligned}
& \xi=-c_{1} x-c_{3} x+c_{5} x^{3}, \\
& \eta=c_{1} y+c_{2} y^{2}+c_{3} x y^{2}+c_{4} x^{2} y^{2}+c_{5}\left(-2 x^{2} y^{2}+x^{3} y^{2}\right) .
\end{aligned}
$$

Thus, the infinitesimal generators of the group of symmetries of (3) are the operators $\Pi_{1}-\Pi_{5}$ described in the statement of Proposition 1, thus having the proposed result.

\section{Optimal System}

Taking into account [22-25], we present in this section the optimal system associated to the symmetry group of (3), which shows a systematic way to classify the invariant solutions. To obtain the optimal system, we should first calculate the corresponding commutator table, which can be obtained from the operator

$$
\left[\Pi_{\alpha}, \Pi_{\beta}\right]=\Pi_{\alpha} \Pi_{\beta}-\Pi_{\beta} \Pi_{\alpha}=\sum_{i=1}^{n}\left(\Pi_{\alpha}\left(\xi_{\beta}^{i}\right)-\Pi_{\beta}\left(\xi_{\alpha}^{i}\right)\right) \frac{\partial}{\partial x^{i}},
$$

where $i=1,2$, with $\alpha, \beta=1, \ldots, 5$, and $\xi_{\alpha}^{i}, \xi_{\beta}^{i}$ are the corresponding coefficients of the infinitesimal operators $\Pi_{\alpha}, \Pi_{\beta}$. After applying the operator (11) to the symmetry group of (3), we obtain the operators that are shown in Table 1.

Now, the next thing is to calculate the adjoint action representation of the symmetries of (3), and to do that, we use Table 1 and the operator

$$
\operatorname{Ad}(\exp (\lambda \Pi)) H=\sum_{n=0}^{\infty} \frac{\lambda^{n}}{n !}(\operatorname{ad}(\Pi))^{n} G \text { for the symmetries }
$$

$\Pi$ and $G$.

Making use of this operator, we can construct Table 2, which shows the adjoint representation for each $\Pi_{i}$.

Proposition 2. The optimal system associated to equation (3) is given by the vector fields

$$
\begin{aligned}
& \Pi_{5}, a_{2} \Pi_{2}, \Pi_{4}, \\
& a_{1} \Pi_{1}+a_{3} \Pi_{3}, \\
& b_{1} \Pi_{4}+\Pi_{5} \text {, } \\
& -a_{3} \Pi_{1}+a_{3} \Pi_{3} \text {, } \\
& a_{3} \Pi_{3}-2 a_{3} b_{12} \Pi_{5}, \\
& \Pi_{2}+b_{17} \Pi_{4} \text {, } \\
& -a_{3} \Pi_{1}+a_{3} \Pi_{3}+\Pi_{5}, \\
& a_{2} \Pi_{2}+a_{3} \Pi_{3}+\left(b_{9}+\frac{a_{2}}{a_{3}}\right) \Pi_{4}, \\
& a_{2} \Pi_{2}+b_{10} \Pi_{4}+\Pi_{5} \text {, } \\
& a_{1} \Pi_{1}+a_{3} \Pi_{3}+b_{2} \Pi_{5} \text {, } \\
& -\Pi_{1}+\Pi_{3}+b_{3} \Pi_{4}, \\
& -2 \Pi_{1}+\Pi_{3}+b_{14} \Pi_{5} \text {, } \\
& a_{1} \Pi_{1}+b_{5} \Pi_{4}+b_{6} \Pi_{5} \text {, } \\
& \Pi_{1}+b_{7} \Pi_{2}+b_{8} \Pi_{5}, \\
& -2 a_{3} \Pi_{1}+a_{3} \Pi_{3}+\Pi_{4}+2 a_{3} b_{13} \Pi_{5} \text {, } \\
& a_{2} \Pi_{2}+a_{3} \Pi_{3}+b_{11} \Pi_{4}-\frac{a_{3} b_{11}}{a_{2}} \Pi_{5}, \\
& a_{1} \Pi_{1}+\Pi_{3}+b_{3} \Pi_{4}+b_{4} \Pi_{5}, \\
& -2 \Pi_{1}+\Pi_{3}+b_{15} \Pi_{4}+b_{16} \Pi_{5} .
\end{aligned}
$$


TABLE 1: Commutators table associated to the symmetry group of (3).

\begin{tabular}{lccccc}
\hline & $\Pi_{1}$ & $\Pi_{2}$ & $\Pi_{3}$ & $\Pi_{4}$ & $\Pi_{5}$ \\
\hline$\Pi_{1}$ & 0 & $\Pi_{2}$ & 0 & $-\Pi_{4}$ & 0 \\
$\Pi_{2}$ & $-\Pi_{2}$ & 0 & 0 & $-2 \Pi_{5}$ \\
$\Pi_{3}$ & 0 & 0 & 0 & $-2 \Pi_{4}$ & $2 \pi_{4}$ \\
$\Pi_{4}$ & $\Pi_{4}$ & 0 & $2 \Pi_{4}$ & 0 & $-2 \Pi_{5}$ \\
$\Pi_{5}$ & $2 \Pi_{5}$ & $-2 \Pi_{4}$ & $2 \Pi_{5}$ & 0 & 0 \\
\hline
\end{tabular}

TABLE 2: Adjoint representation of the symmetry group of (3).

\begin{tabular}{lccccc}
\hline $\operatorname{adj}[]$, & $\Pi_{1}$ & $\Pi_{2}$ & $\Pi_{3}$ & $\Pi_{4}$ & $\Pi_{5}$ \\
\hline$\Pi_{1}$ & $\Pi_{1}$ & $e^{-\lambda} \Pi_{2}$ & $\Pi_{3}$ & $e^{\lambda} \Pi_{4}$ & $\Pi_{4}$ \\
$\Pi_{2}$ & $\Pi_{1}+\lambda \Pi_{2}$ & $\Pi_{2}$ & $\Pi_{3}$ & $\Pi_{5}$ \\
$\Pi_{3}$ & $\Pi_{1}$ & $\Pi_{2}$ & $\Pi_{3}$ & $e^{2 \lambda} \Pi_{4}$ & $\Pi_{3}-2 \lambda \Pi_{4}$ \\
$\Pi_{4}$ & $\Pi_{1}-\lambda \Pi_{4}$ & $\Pi_{2}$ & $\Pi_{3}-2 \lambda \Pi_{4}$ & $\Pi_{4}$ & $e_{5}$ \\
$\Pi_{5}$ & $\Pi_{1}-2 \lambda \Pi_{5}$ & $\Pi_{2}+2 \lambda \Pi_{4}$ & $\Pi_{3}-2 \lambda \Pi_{5}$ & $\Pi_{5}$ & $\Pi_{5}$ \\
\hline
\end{tabular}

Proof. To calculate the optimal system, we start with the generators of symmetries (4) and a generic nonzero vector. Let

$$
G=a_{1} \Pi_{1}+a_{2} \Pi_{2}+a_{3} \Pi_{3}+a_{4} \Pi_{4}+a_{5} \Pi_{5} .
$$

The objective is to simplify as many coefficients, $a_{i}$, as possible, through maps adjoint to $G$, using Table 2 .

(1) Assuming $a_{5}=1$ in (14), we have that $G=a_{1} \Pi_{1}+a_{2} \Pi_{2}+a_{3} \Pi_{3}+a_{4} \Pi_{4}+\Pi_{5}$. Applying the adjoint operator to $\left(\Pi_{1}, G\right)$ and $\left(\Pi_{3}, G\right)$, we do not have any reduction; on the other hand, applying the adjoint operator to $\left(\Pi_{2}, G\right)$, we get

$$
\begin{aligned}
G_{1}= & \operatorname{Ad}\left(\exp \left(\lambda_{1} \Pi_{2}\right)\right) G=a_{1} \Pi_{1}+\Pi_{2}\left(a_{2}+a_{1} \lambda_{1}\right) \\
& +a_{3} \Pi_{3}+\Pi_{4}\left(a_{4}-2 \lambda_{1}\right)+\Pi_{5} .
\end{aligned}
$$

(1.1) Case $a_{1} \neq 0$. Using $\lambda_{1}=\left(-a_{2} / a_{1}\right) a_{1} \neq 0$, in (15), $\Pi_{2}$ is eliminated, therefore $G_{1}=a_{1} \Pi_{1}+a_{3} \Pi_{3}$ $+b_{1} \Pi_{4}+\Pi_{5}$, where $b_{1}=a_{4}+\left(2 a_{2} / a_{1}\right)$. Now, applying the adjoint operator to $\left(\Pi_{4}, G_{1}\right)$, we get $G_{2}=$ $\operatorname{Ad}\left(\exp \left(\lambda_{2} \Pi_{4}\right)\right) G_{1}=a_{1} \Pi_{1}+a_{3} \Pi_{3}+\left(b_{1}-\lambda_{2}\left(a_{1}+\right.\right.$ $\left.\left.2 a_{3}\right)\right) \Pi_{4}+\Pi_{5}$.

(1.1.A) Case $a_{1}+2 a_{3} \neq 0$. Using $\lambda_{2}=\left(b_{1} / a_{1}+2 a_{3}\right)$, with $a_{1}+2 a_{3} \neq 0$, eliminated is $\Pi_{4}$, and then $G_{2}=a_{1} \Pi_{1}+a_{3} \Pi_{3}+\Pi_{5}$. Applying the adjoint operator to $\left(\Pi_{5}, G_{2}\right)$, we get

$$
\begin{aligned}
G_{3}= & \operatorname{Ad}\left(\exp \left(\lambda_{3} \Pi_{5}\right)\right) G_{2}=a_{1} \Pi_{1}+a_{3} \Pi_{3} \\
& +\left(1-2 \lambda_{3}\left(a_{1}+a_{3}\right)\right) \Pi_{5} .
\end{aligned}
$$

(1.1.A. $\left.A_{1}\right)$ Case $a_{1}+a_{3} \neq 0$. Using $\lambda_{3}=\left(1 / 2\left(a_{1}+\right.\right.$ $\left.\left.a_{3}\right)\right)$ ), with $a_{1}+a_{3} \neq 0$, in (16), $\Pi_{5}$ is eliminated, therefore $G_{3}=a_{1} \Pi_{1}+a_{3} \Pi_{3}$. Then, we have the first element of the optimal system.

$$
G_{3}=a_{1} \Pi_{1}+a_{3} \Pi_{3},
$$

with $a_{1} \neq 0, a_{1}+2 a_{3} \neq 0$, and $a_{1}+a_{3} \neq 0$. This is how the first reduction of the generic element (14) ends.

(1.1.A. $\left.A_{2}\right)$ Case $a_{1}+a_{3}=0$. We get $G_{3}=$ $-a_{3} \Pi_{1}+a_{3} \Pi_{3}+\Pi_{5}$. Then, we have the other element of the optimal system.

$$
G_{3}=-a_{3} \Pi_{1}+a_{3} \Pi_{3}+\Pi_{5},
$$

with $a_{3} \neq 0$. This is how the other reduction of the generic element (14) ends.

(1.1.B) Case $a_{1}+2 a_{3}=0$. We get $G_{2}=-2 a_{3} \Pi_{1}$ $+a_{3} \Pi_{3}+b_{1} \Pi_{4}+\Pi_{5}$. Applying the adjoint operator to $\left(\Pi_{5}, G_{2}\right)$, we have

$$
\begin{aligned}
G_{19}= & \operatorname{Ad}\left(\exp \left(\lambda_{19} \Pi_{5}\right)\right) G_{2}=-2 a_{3} \Pi_{1}+a_{3} \Pi_{3}+b_{1} \Pi_{4} \\
& +\left(1+4 a_{3} \lambda_{19}\right) \Pi_{5} .
\end{aligned}
$$

(1.1.B.1) Case $a_{3} \neq 0$. Using $\lambda_{19}=\left(-1 / 4 a_{3}\right)$, with $a_{3} \neq 0$, in (19), $\Pi_{5}$ is eliminated, therefore $G_{19}=-2 a_{3} \Pi_{1}+a_{3} \Pi_{3}+b_{1} \Pi_{4}$. Then, we have the other element of the optimal system.

$$
G_{19}=-2 a_{3} \Pi_{1}+a_{3} \Pi_{3}+b_{1} \Pi_{4},
$$

with $a_{1} \neq 0, a_{1}+2 a_{3} \neq 0$, and $a_{1}+a_{3} \neq 0$. This is how the other reduction of the generic element (14) ends.

(1.1.B.2) Case $a_{3}=0$. We get $G_{19}=b_{1} \Pi_{4}+\Pi_{5}$. Then, we have the other element of the optimal system.

$$
G_{19}=b_{1} \Pi_{4}+\Pi_{5} .
$$

This is how the other reduction of the generic element (14) ends.

(1.2) Case $a_{1}=0$. We get $G_{1}=a_{2} \Pi_{2}+$ $a_{3} \Pi_{3}+\left(a_{4}-2 \lambda_{1}\right) \Pi_{4}+\Pi_{5}$, using $\lambda_{1}=\left(a_{4} / 2\right)$, then $\Pi_{4}$ is eliminated, and then $G_{1}=a_{2} \Pi_{2}+a_{3} \Pi_{3}+\Pi_{5}$. Now, applying the adjoint operator to $\left(\Pi_{4}, G_{1}\right)$, we have $\quad G_{16}=\operatorname{Ad}\left(\exp \left(\lambda_{16} \Pi_{4}\right)\right) G_{1}=a_{2} \Pi_{2}+a_{3}$ 
$\Pi_{3}-2 a_{3} \lambda_{16} \Pi_{4}+\Pi_{5}$. It is clear that we do not have any reduction.

(1.2.1) Case $a_{3} \neq 0$. Then, using $\lambda_{16}=\left(-b_{9} / 2 a_{3}\right)$, with $a_{3} \neq 0$, we get $G_{16}=a_{2} \Pi_{2}+a_{3} \Pi_{3}+b_{9} \Pi_{4}+\Pi_{5}$. Applying the adjoint operator to $\left(\Pi_{5}, G_{16}\right)$, we have

$$
\begin{aligned}
G_{17}= & \operatorname{Ad}\left(\exp \left(\lambda_{17} \Pi_{5}\right)\right) G_{16}=a_{2} \Pi_{2}+a_{3} \Pi_{3} \\
& +\left(b_{9}+2 a_{2} \lambda_{17}\right) \Pi_{4}+\left(1-2 a_{3} \lambda_{17}\right) \Pi_{5} .
\end{aligned}
$$

Using $\lambda_{17}=\left(1 / 2 a_{3}\right)$, with $a_{3} \neq 0$, in (22), $\Pi_{5}$ is eliminated, therefore $G_{17}=a_{2} \Pi_{2}+a_{3} \Pi_{3}+$ $\left(b_{9}+\left(a_{2} / a_{3}\right)\right) \Pi_{4}$. Then, we have the other element of the optimal system.

$$
G_{17}=a_{2} \Pi_{2}+a_{3} \Pi_{3}+\left(b_{9}+\frac{a_{2}}{a_{3}}\right) \Pi_{4}, \quad \text { with } a_{3} \neq 0 \text {. }
$$

(1.2.2) Case $a_{3}=0$. We get $G_{16}=a_{2} \Pi_{2}+\Pi_{5}$. Applying the adjoint operator to $\left(\Pi_{5}, G_{16}\right)$, we have

$G_{18}=\operatorname{Ad}\left(\exp \left(\lambda_{18} \Pi_{5}\right)\right) G_{16}=a_{2} \Pi_{2}+2 a_{2} \lambda_{18} \Pi_{4}+\Pi_{5}$.

It is clear that we do not have any reduction.

(1.2.2.A) Case $a_{2} \neq 0$. Then, using $\lambda_{18}=\left(-b_{10} / 2 a_{2}\right)$, with $a_{2} \neq 0$, in (24), we get $G_{18}=a_{2} \Pi_{2}+b_{10} \Pi_{4}+\Pi_{5}$. Then, we have the other element of the optimal system.

$$
G_{18}=a_{2} \Pi_{2}+b_{10} \Pi_{4}+\Pi_{5}, \quad \text { with } a_{2} \neq 0 .
$$

(1.2.2.B) Case $a_{2}=0$. Then, we get $G_{18}=\Pi_{5}$, hence we have the other element of the optimal system.

$$
G_{18}=\Pi_{5}
$$

(2) Assuming $a_{5}=0$ and $a_{4}=1$ in (14), we have that $G=a_{1} \Pi_{1}+a_{2} \Pi_{2}+a_{3} \Pi_{3}+\Pi_{4}$. Applying the adjoint operator to $\left(\Pi_{1}, G\right)$ and $\left(\Pi_{3}, G\right)$, we do not have any reduction; on the other hand, applying the adjoint operator to $\left(\Pi_{2}, G\right)$, we get

$$
\begin{aligned}
G_{4}= & \operatorname{Ad}\left(\exp \left(\lambda_{4} \Pi_{2}\right)\right) G=a_{1} \Pi_{1}+\left(a_{2}+a_{1} \lambda_{4}\right) \Pi_{2} \\
& +a_{3} \Pi_{3}+\Pi_{4} .
\end{aligned}
$$

(2.1) Case $a_{1} \neq 0$. Using $\lambda_{4}=\left(-a_{2} / a_{1}\right)$ with $a_{1} \neq 0$, in (27), $\Pi_{2}$ is eliminated, therefore $G_{4}=a_{1} \Pi_{1}$ $+a_{3} \Pi_{3}+\Pi_{4}$. Now, applying the adjoint operator to $\left(\Pi_{4}, G_{4}\right)$, we get $G_{5}=\operatorname{Ad}\left(\exp \left(\lambda_{5} \Pi_{4}\right)\right) G_{4}=a_{1} \Pi_{1}+$ $a_{3} \Pi_{3}+\left(1-\lambda_{5}\left(a_{1}+2 a_{3}\right)\right) \Pi_{4}$.

(2.1.A) Case $a_{1}+2 a_{3} \neq 0$. Using $\lambda_{5}=\left(1 / a_{1}+2 a_{3}\right)$, with $a_{1}+2 a_{3} \neq 0$, eliminated is $\Pi_{4}$, then $G_{5}=a_{1} \Pi_{1}+a_{3} \Pi_{3}$. Applying the adjoint operator to $\left(\Pi_{5}, G_{5}\right)$, we get

$$
\begin{aligned}
G_{6}= & \operatorname{Ad}\left(\exp \left(\lambda_{6} \Pi_{5}\right)\right) G_{5}=a_{1} \Pi_{1}+a_{3} \Pi_{3} \\
& \left.-2 \lambda_{6}\left(a_{1}+a_{3}\right)\right) \Pi_{5} .
\end{aligned}
$$

It is clear that we do not have any reduction.
(2.1.A. $\left.A_{1}\right)$ Case $a_{1}+a_{3} \neq 0$. Then, substituting $\lambda_{6}=$ $\left(-b_{2} / 2\left(a_{1}+a_{3}\right)\right)$ with $a_{1}+a_{3} \neq 0$, we have the other element of the optimal system

$$
G_{6}=a_{1} \Pi_{1}+a_{3} \Pi_{3}+b_{2} \Pi_{5}
$$

with $a_{1} \neq 0, a_{1}+2 a_{3} \neq 0$, and $a_{1}+a_{3} \neq 0$. This is how the other reduction of the generic element (14) ends.

(2.1.A. $\left.A_{2}\right)$ Case $a_{1}+a_{3}=0$. We get $G_{6}=$ $-a_{3} \Pi_{1}+a_{3} \Pi_{3}$, and then we have the other element of the optimal system,

$$
G_{6}=-a_{3} \Pi_{1}+a_{3} \Pi_{3}
$$

with $a_{1}, a_{3} \neq 0$ and $a_{1}+2 a_{3} \neq 0, a_{1}=-a_{3}$. This is how the other reduction of the generic element (14) ends.

(2.1.B) Case $a_{1}+2 a_{3}=0$. We get $G_{5}=-2 a_{3} \Pi_{1}$ $+a_{3} \Pi_{3}+\Pi_{4}$. Applying the adjoint operator to $\left(\Pi_{5}, G_{5}\right)$, we have

$$
\begin{aligned}
G_{23}= & \operatorname{Ad}\left(\exp \left(\lambda_{23} \Pi_{5}\right)\right) G_{5}=-2 a_{3} \Pi_{1}+a_{3} \Pi_{3}+\Pi_{4} \\
& +2 a_{3} \lambda_{23} \Pi_{5} .
\end{aligned}
$$

It is clear that we do not have any reduction; it is also clear that $a_{1} \neq 0$ and then $a_{3} \neq 0$; then, substituting $\lambda_{23}=b_{13}$, we have the other element of the optimal system

$$
G_{23}=-2 a_{3} \Pi_{1}+a_{3} \Pi_{3}+\Pi_{4}+2 a_{3} b_{13} \Pi_{5},
$$

with $a_{3} \neq 0 \quad y \quad a_{1}+2 a_{3}=0$. This is how the other reduction of the generic element (14) ends.

(2.2) Case $a_{1}=0$. We get $G_{4}=a_{2} \Pi_{2}+a_{3} \Pi_{3}+\Pi_{4}$. Now, applying the adjoint operator to $\left(\Pi_{4}, G_{4}\right)$, we have $\quad G_{20}=\operatorname{Ad}\left(\exp \left(\lambda_{20} \Pi_{4}\right)\right) G_{4}=a_{2} \Pi_{2}+a_{3} \Pi_{3}+$ $\left(1-2 a_{3} \lambda_{20}\right) \Pi_{4}$.

(2.2.A) Case $a_{3} \neq 0$. Using $\lambda_{20}=\left(1 / 2 a_{3}\right)$, with $a_{3} \neq 0, \Pi_{4}$ is eliminated, then $G_{20}=a_{2} \Pi_{2}+a_{3} \Pi_{3}$. Applying the adjoint operator to $\left(\Pi_{5}, G_{20}\right)$, we get

$$
\begin{aligned}
G_{21}= & \operatorname{Ad}\left(\exp \left(\lambda_{21} \Pi_{5}\right)\right) G_{20}=a_{2} \Pi_{2}+a_{3} \Pi_{3} \\
& +2 a_{2} \lambda_{21} \Pi_{4}-2 a_{3} \lambda_{21} \Pi_{5} .
\end{aligned}
$$

It is clear that we do not have any reduction. (2.2.A.1) Case $a_{2} \neq 0$. Then, substituting $\lambda_{21}=$ $\left(+b_{11} / 2 a_{2}\right)$ with $a_{2} \neq 0$, we have other element of the optimal system

$$
G_{21}=a_{2} \Pi_{2}+a_{3} \Pi_{3}+b_{11} \Pi_{4}-\frac{a_{3} b_{11}}{a_{2}} \Pi_{5},
$$

with $a_{2}, a_{3} \neq 0$. This is how the other reduction of the generic element (14) ends.

(2.2.A.2) Case $a_{2}=0$. We get $G_{21}=$ $a_{3} \Pi_{3}-2 a_{3} \lambda_{21} \Pi_{5}$; we do not have any reduction; then, using $\lambda_{21}=b_{12}$, we have the other element of the optimal system 


$$
G_{21}=a_{3} \Pi_{3}-2 a_{3} b_{12} \Pi_{5}, \quad \text { with } a_{2} \neq 0 .
$$

This is how the other reduction of the generic element (14) ends.

(2.2.B) Case $a_{3}=0$. We get $G_{20}=a_{2} \Pi_{2}+\Pi_{4}$. Applying the adjoint operator to $\left(\Pi_{5}, G_{20}\right)$, we get

$G_{22}=\operatorname{Ad}\left(\exp \left(\lambda_{22} \Pi_{5}\right)\right) G_{20}=a_{2} \Pi_{2}+\left(1-2 a_{2} \lambda_{22}\right) \Pi_{4}$.

(2.2.B.1) Case $a_{2} \neq 0$. Using $\lambda_{22}=\left(1 / 2 a_{2}\right)$ with $a_{2} \neq 0, \Pi_{4}$ is eliminated, then we have the other element of the optimal system

$$
G_{22}=a_{2} \Pi_{2}, \quad \text { with } a_{2} \neq 0 .
$$

This is how the other reduction of the generic element (14) ends.

(2.2.B.2) Case $a_{2}=0$. We get $G_{22}=\Pi_{4}$, and then we have the other element of the optimal system

$$
G_{22}=\Pi_{4}
$$

This is how the other reduction of the generic element (14) ends.

(3) Following a procedure analogous to the previous one and analyzing the respective cases for $a_{4}=a_{5}=0$, $a_{3}=1$ in (14); $a_{3}=a_{4}=a_{5}=0, a_{2}=1$ in (14) and $a_{2}=a_{3}=a_{4}=a_{5}=0, a_{1}=1$ in (14); we can reduce and obtain all the elements presented for the optimal system.

\section{Invariant Solutions by Some Generators of the Optimal System}

In this section, we characterize invariant solutions taking into account all operators that generate the optimal system presented in Proposition 2. For this purpose, we use the method of invariant curve condition [23] (presented in Section 4.3), which is given by the following equation:

$$
Q\left(x, y, y_{x}\right)=\eta-y_{x} \xi=0 \text {. }
$$

Using the element $\Pi_{5}$ from Proposition 2, under the condition (39), we obtain that $Q=\eta_{5}-y_{x} \xi_{5}=0$, which implies $\left(-2 x^{2} y+x^{3} y^{2}\right)-y_{x}\left(x^{3}\right)=0$; then, solving this ODE, we have $y(x)=\left(1 / x\left(c_{1} x+1\right)\right)$, where $c$ is an arbitrary constant, which is an invariant solution for (3); using an analogous procedure with all of the elements of the optimal system (Proposition 2), we obtain both implicit and explicit invariant solutions that are shown in Table 3 , with $c$ being a constant.

\section{Variational Symmetries and Conserved Quantities}

In this section, we present the variational symmetries of (3) and we are going to use them to define conservation laws via Noether's theorem [26]. First of all, we are going to determine the Lagrangian using the Jacobi last multiplier method, presented by Nucci in [27], and for this reason, we are urged to calculate the inverse of the determinant $\Delta$,

$$
\Delta=\left|\begin{array}{ccc}
x & y_{x} & y_{x x} \\
\Pi_{2, x} & \Pi_{2, y} & \Pi_{2}^{(1)} \\
\Pi_{4, x} & \Pi_{4, y} & \Pi_{4}^{(1)}
\end{array}\right|=\left|\begin{array}{ccc}
x & y_{x} & y_{x x} \\
0 & y^{2} & 2 y y_{x} \\
0 & x^{2} y^{2} & 2 x y^{2}+2 x^{2} y y_{x}
\end{array}\right|,
$$

where $\Pi_{2, x}, \Pi_{2, y}, \Pi_{4, x}$, and $\Pi_{4, y}$ are the components of the symmetries $\Pi_{2}, \Pi_{4}$ shown in the Proposition 4 and $\Pi_{2}^{(1)}, \Pi_{4}^{(1)}$ are its first prolongations. Then, we get $\Delta=2 x y^{4}$ which implies that $M=(1 / \Delta)=\left(x^{-1} y^{-4} / 2\right)$. Now, from [27], we know that $M$ can also be written as $M=L_{y_{x} y_{x}}$ which means that $L_{y_{x} y_{x}}=\left(x^{-1} y^{-4} / 2\right)$; then, integrating twice with respect to $y_{x}$, we obtain the Lagrangian,

$$
L\left(x, y, y_{x}\right)=\frac{y_{x}^{2}}{4 x y^{4}}+y_{x} f_{1}(x, y)+f_{2}(x, y),
$$

where $f_{1}, f_{2}$ are arbitrary functions. From the preceding expression, we can consider $f_{1}=f_{2}=0$. It is possible to find more Lagrangians for (3) by considering other vector fields given in the Proposition 4 . We then calculate

$$
\begin{aligned}
& \xi(x, y) L_{x}+\xi_{x}(x, y) L+\eta(x, y) L_{y}+\eta_{[x]}(x, y) L_{y_{x}} \\
& =D_{x}[f(x, y)]
\end{aligned}
$$

using (41) and (8). Thus, we get

$$
\begin{aligned}
& \xi\left(-\frac{y_{x}^{2} x^{-2} y^{-4}}{4}\right)+\xi_{x}\left(\frac{y_{x}^{2} x^{-1} y^{-4}}{4}\right)+\eta\left(-x^{-1} y^{-5} y_{x}^{2}\right) \\
& \quad+\left(\eta_{x}+\left(\eta_{y}-\xi_{x}\right) y_{x}-\xi_{y} y_{x}^{2}\right)\left(\frac{x^{-1} y^{-4} y_{x}}{2}\right)-f_{x}-y_{x} f_{y}=0 .
\end{aligned}
$$

From the preceding expression, rearranging and associating terms with respect to $1, y_{x}, y_{x}^{2}$ and $y_{x}^{3}$, we obtain the following determinant equations:

$$
\begin{array}{r}
\xi_{y}=f_{x}=0, \\
\frac{x^{-1} y^{-4}}{2} \eta_{x}-f_{y}=0, \\
-y \xi-4 x \eta-2 x y \xi_{x}+2 x y \eta_{y}=0 .
\end{array}
$$

Solving the preceding system for $\xi, \eta$ and $f$, we obtain the infinitesimal generators of Noether's symmetries

$$
\begin{aligned}
\eta & =-2 a_{1} y x^{2}-a_{2} y^{2} x^{2}+a_{4} y^{2}+a_{5} y, \\
\xi & =-\frac{4 a_{5} x^{2}}{5}-\frac{8 a_{1} x^{4}}{9} \\
f(y) & =a_{1} y^{-2}+a_{2} y^{-1}+a_{3} .
\end{aligned}
$$

with $a_{1}, a_{2}, a_{3}, a_{4}$, and $a_{5}$ arbitrary constants. Then, the Noether symmetry group or variational symmetries are 


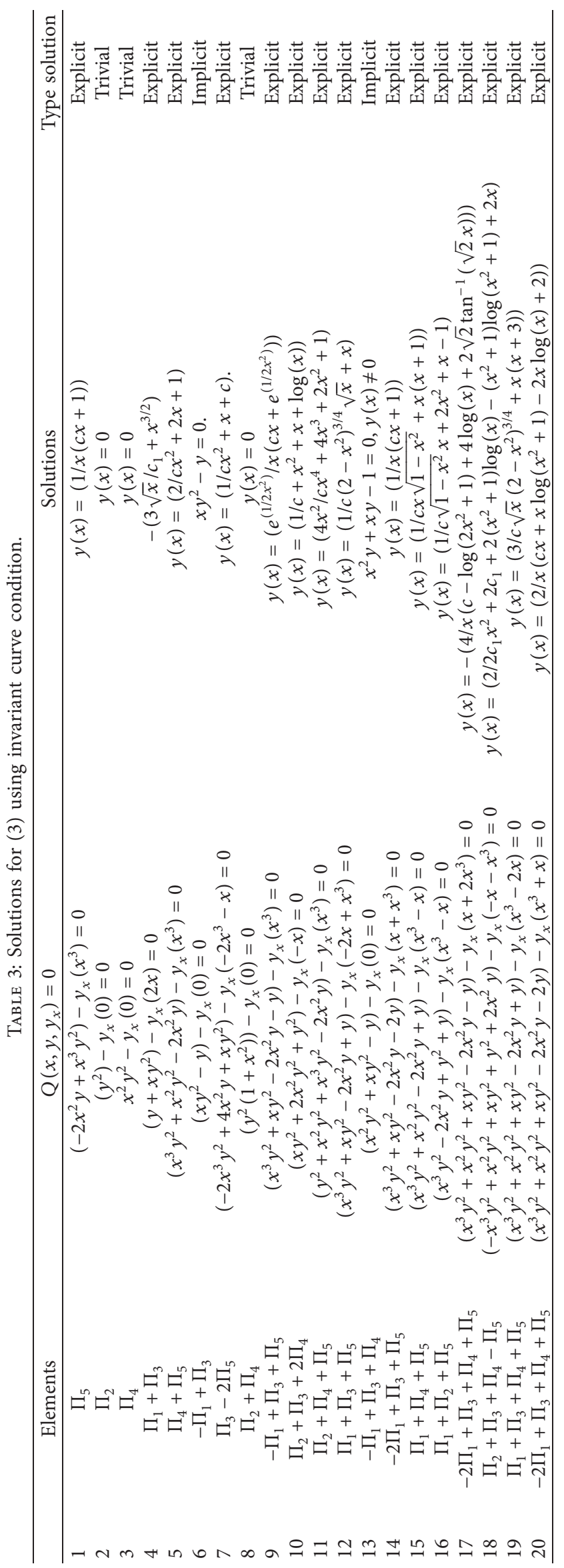




$$
\begin{aligned}
& V_{1}=-\frac{8 x^{4}}{9} \frac{\partial}{\partial x}-2 x^{2} y \frac{\partial}{\partial y}, \\
& V_{2}=-x^{2} y^{2} \frac{\partial}{\partial y} \\
& V_{3}=y^{2} \frac{\partial}{\partial y} \\
& V_{4}=-\frac{4 x^{2}}{5} \frac{\partial}{\partial x}+y \frac{\partial}{\partial y} .
\end{aligned}
$$

Remark 1. Note that $V_{2}=-\Pi_{4}$ and $V_{3}=\Pi_{2}$, thus the symmetries of equation (3) have two variational symmetries. According to [28], in order to obtain the conserved quantities or conservation laws, we should solve

$$
I=\left(X y_{x}-Y\right) L_{y_{x}}-X L+f
$$

using (41), (45), and (46). Therefore, the conserved quantities are given by

$$
\begin{aligned}
& I_{1}=-\frac{2 x^{3} y^{-4} y_{x}^{2}}{9}+x y^{-3} y_{x}+a_{1} y^{-2}+a_{2} y^{-1}+a_{3}, \\
& I_{2}=\frac{x y^{-2} y_{x}}{2}+a_{1} y^{-2}+a_{2} y^{-1}+a_{3}, \\
& I_{3}=-\frac{x^{-1} y^{-1} y_{x}}{2}+a_{1} y^{-2}+a_{2} y^{-1}+a_{3}, \\
& I_{4}=-\frac{x y^{-4} y_{x}^{2}}{5}-\frac{x^{-1} y^{-3} y_{x}}{2}+a_{1} y^{-2}+a_{2} y^{-1}+a_{3} .
\end{aligned}
$$

\section{Nonlinear Self-Adjointness}

In this section, we present the main definitions in the $\mathrm{N}$. Ibragimov's approach to nonlinear self-adjointness of differential equations adopted to our specific case. For further details, the interested reader is directed to [29-31].

Consider second-order differential equation

$$
\mathfrak{F}\left(x, y, y_{(1)}, y_{(2)}, \ldots, y_{(s)}\right)=0,
$$

with independent variables $x$ and a dependent variable $y$, where $y_{(1)}, y_{(2)}, \ldots, y_{(s)}$ denote the collection of $1,2, \ldots, s$-th order derivatives of $y$.

Definition 1. Let $\mathfrak{F}$ be a differential function and $\nu=v(x)$ be the new dependent variable, known as the adjoint variable or nonlocal variable [31]. The formal Lagrangian for $\mathfrak{F}=0$ is the differential function defined by

$$
\mathfrak{L}:=\nu \mathfrak{F}
$$

Definition 2. Let $\mathfrak{F}$ be a differential function and for the differential equation (49), denoted by $\mathfrak{F}[y]=0$, we define the adjoint differential function to $\mathfrak{F}$ by

$$
\mathfrak{F}^{*}:=\frac{\delta \mathfrak{Q}}{\delta y}
$$

and the adjoint differential equation by

$$
\mathfrak{F}^{*}[y, \nu]=0,
$$

where the Euler operator

$$
\frac{\delta}{\delta y}=\frac{\partial}{\partial y}+\sum_{m=1}^{\infty}(-1)^{m} D_{x_{i}}, \ldots, D_{x_{i}, m}, \frac{\partial}{\partial y_{x_{i_{1}} x_{i_{2}} \cdots x_{i_{m}}}},
$$

and $D_{x_{i}}$ is the total derivative operator with respect to $x_{i}$ defined by

$$
D_{x_{i}}=\partial_{x_{i}}+y_{x_{i}} \partial_{y}+y_{x_{i} x_{j}} \partial_{y_{x_{j}}}+\cdots+y_{x_{i} x_{i_{1}} x_{i_{2}}, \ldots, x_{i_{n}}} \partial_{y_{x_{i_{1}}} x_{i_{2}} \cdots x_{i_{n}}} \cdots
$$

Definition 3. The differential equation (40) is said to be nonlinearly self-adjoint if there exists a substitution

$$
v=\phi(x, y) \neq 0
$$

such that

$$
\left.\widetilde{\mathfrak{F}}^{*}\right|_{\nu=\phi(x, y)}=\lambda \mathfrak{F}
$$

for some undetermined coefficients $\lambda=\lambda(x, y, \ldots)$. If $v=$ $\phi(y)$ in the two previous expressions, equation (49) is called quasi-self-adjoint. If $v=y$, we say that equation (49) is strictly self-adjoint.

Now, we shall obtain the adjoint equation to equation (3). For this purpose, we write (3) in the form (49), where

$$
\mathfrak{F}:=y_{x x}-2 y_{x}^{2} y^{-1}-x^{-1} y_{x}-x^{-1} y^{2}=0 .
$$
by

Then, the corresponding formal Lagrangian (50) is given

$$
\mathfrak{L}:=\nu\left(y_{x x}-2 y_{x}^{2} y^{-1}-x^{-1} y_{x}-x^{-1} y^{2}\right)=0,
$$

and the Euler operator (53) assumes the following form:

$$
\frac{\delta \mathfrak{Q}}{\delta y}=\frac{\partial \mathfrak{Q}}{\partial y}-D_{x} \frac{\partial \mathfrak{Q}}{\partial y_{x}}+D_{x}^{2} \frac{\partial \mathfrak{Q}}{\partial y_{x x}} .
$$

We calculate explicitly the Euler operator previously applied to $\mathbf{Q}$ determined by (58). In this way, we obtain the adjoint equation (52) to (3):

$$
\begin{aligned}
\mathfrak{F}^{*}= & -v\left(-2 y_{x}^{2} y^{-2}+4 y_{x x} y^{-1}-2 x^{-1} y-x^{-2}\right) \\
& +v_{x}\left(4 y_{x} y^{-1}+x^{-1}\right)+v_{x x}=0 .
\end{aligned}
$$

The main result in this section can be stated as follows.

Proposition 3. Equation (3) is nonlinearly self-adjoint, with the substitution given by

$$
\phi(x, y)=y^{-2}\left(k_{2} x^{(1 / 2(1+\sqrt{5}))}+k_{1} x^{(1 / 2(1-\sqrt{5}))}\right),
$$

where $k_{1}, k_{2}$ are the arbitrary constants. 
Proof. Substituting in (60), and then in (56), $v=\phi(x, y)$ and its respective derivatives, and comparing the corresponding coefficients, we get the following five equations:

$$
\begin{aligned}
-\phi_{y} & =\lambda, \\
4 y^{-1} \phi+2 \phi_{y} & =0, \\
4 y^{-1} \phi_{x}+2 \phi_{x y} & =0, \\
-2 y^{-2} \phi+2 y^{-1} \phi_{y}+\phi_{y y} & =0, \\
\phi\left(-2 x^{-1} y-x^{-2}\right)+\phi_{x x}-x^{-1} y^{2} \phi_{y} & =0 .
\end{aligned}
$$

We observe that equation (62c) is obtained from equation (62b) by differentiation with respect to $x$. Therefore, we have to study only equations (62b), (62d), and (62e). Solving for $\phi$ in (62b), we obtain

$$
\phi(x, y)=c_{1}(x) y^{-2},
$$

where $c_{1}(x)$ is the arbitrary function. Using (63) in (62e), we get $-\left(c_{1}(x) / x^{2}\right)+c_{1, x x}(x)=0$, thus solving for $c_{1}(x)$, we have $\quad c_{1}(x)=k_{2} x^{(1 / 2(1+\sqrt{5}))}+k_{1} x^{(1 / 2(1-\sqrt{5}))}$; then, substituting in (63), the statement in the theorem is obtained.

\section{Conservation Laws}

In this section, we shall establish some conservation laws for equation (3) using the conservation theorem of $\mathrm{N}$ [31]. Since equation (3) is of second order, the formal Lagrangian contains derivatives up to order two. Thus, the general formula in [31] for the component of the conserved vector is reduced to

$$
C^{x}=W^{j}\left[\frac{\partial \mathfrak{Q}}{\partial_{y_{x}}}-D_{x}\left(\frac{\partial \mathfrak{Q}}{\partial_{y_{x x}}}\right)\right]+D_{x}\left[W^{j}\right]\left[\frac{\partial \mathfrak{Q}}{\partial_{y_{x x}}}\right],
$$

where

$$
W^{j}=\eta^{j}-\xi^{j} y_{x}
$$

where $j=1, \ldots, 5$ is the formal Lagrangian (58),

$$
\mathfrak{Q}:=v\left(y_{x x}-2 y_{x}^{2} y^{-1}-x^{-1} y_{x}-x^{-1} y^{2}\right),
$$

and $\eta^{j}, \xi^{j}$ are the infinitesimals of a Lie point symmetry admitted by equation (3), given in (4). Using (3), (4), and (3) in (64), we obtain the following conservation vectors for each symmetry stated in (4).

$$
\begin{aligned}
& C_{1}^{x}=v\left(y_{x}(-2)+y_{x}^{2}\left(-4 x y^{-1}+2 y^{-1}\right)-x^{-1} y+y^{2}\right)-v_{x}\left(y+x y_{x}\right), \\
& C_{2}^{x}=v\left(y_{x}(-2 y)-2 x^{-1} y^{2}\right)-v_{x}\left(y^{2}\right), \\
& C_{3}^{x}=v\left(y_{x}(-2 x y)+y_{x}^{2}\left(-2 x y^{-1}-1\right)+y^{2}\right)-v_{x}\left(x y^{2}+x y_{x}\right), \\
& C_{4}^{x}=v\left(y_{x}\left(-2 x^{2} y\right)+x y^{2}\right)-v_{x}\left(x^{2} y^{2}\right), \\
& C_{5}^{x}=v\left(y_{x}\left(-13 x^{2}+6 x^{3} y\right)+y_{x}^{2}\left(4 x^{3} y^{-1}-2 x^{3}\right)-6 x y+3 x^{2} y^{2}\right)-v_{x}\left(-2 x^{2} y+x^{3} y^{2}-x^{3} y_{x}\right),
\end{aligned}
$$

where

$$
\begin{aligned}
v & =y^{-2}\left(k_{2} x^{(1 / 2(1+\sqrt{5}))}+k_{1} x^{(1 / 2(1-\sqrt{5}))}\right), \\
v_{x} & =y^{-2}\left(\frac{k_{2} x^{(1 / 2(1+\sqrt{5}))-1}}{2(1+\sqrt{5})}+\frac{k_{1} x^{(1 / 2(1-\sqrt{5}))-1}}{2(1-\sqrt{5})}\right) .
\end{aligned}
$$

\section{Classification of Lie Algebra}

Generically, a finite-dimensional Lie algebra in a field of characteristic 0 is classified by the Levi's theorem, which states that any finite-dimensional Lie algebra can be written as a semidirect product of a semisimple Lie algebra and a solvable Lie algebra; the solvable Lie algebra is the radical of that algebra. In other words, there exist two important classes of Lie algebras, the solvable and the semisimple. In each class mentioned above, there are some particular classes that have other classifications, for example, in the solvable one, we have the nilpotent Lie algebra.
According the Lie group symmetry of generators given in Table 1, we have a five-dimensional Lie algebra. First of all, we remember some basic criteria to classify a Lie algebra. In the case of solvable and semisimple Lie algebra, we will denote $K(\cdot, \cdot)$ as the Cartan-Killing form. The next propositions can be found in [32].

Proposition 4. (Cartan's theorem). A Lie algebra is semisimple if and only if its Cartan-Killing form is nondegenerate.

Proposition 5. A Lie subalgebra $\mathfrak{g}$ is solvable if and only if $K(X, Y)=0$ for all $X \in[\mathfrak{g}, \mathfrak{g}]$ and $Y \in \mathfrak{g}$. Another way to write that is $K(\mathfrak{g},[\mathfrak{g}, \mathfrak{g}])=0$.

We also need the next statements to make the classification.

Definition 4. Let $\mathfrak{g}$ be a finite-dimensional Lie algebra over an arbitrary field $k$. Choose a basis $e_{j}, 1 \leq i \leq n$, in $\mathfrak{g}$ where $n=\operatorname{dim} g$ and set $\left[e_{i}, e_{j}\right]=C_{i j}^{k} e_{k}$. Then, the coefficients $C_{i j}^{k}$ are called structure constants. 
Proposition 6. Let $\mathfrak{g}_{1}$ and $\mathfrak{g}_{2}$ be two Lie algebras of dimension $n$. Suppose each has a basis with respect to which the structure constants are the same. Then, $\mathfrak{g}_{1}$ and $\mathfrak{g}_{2}$ are isomorphic.

Let $\mathfrak{g}$ be the Lie algebra related to the symmetry group of infinitesimal generators of equation (1) as stated by the table of the commutators; it is enough to consider the next relations: $\left[\Pi_{1}, \Pi_{2}\right]=\Pi_{2},\left[\Pi_{1}, \Pi_{4}\right]=-\Pi_{4},\left[\Pi_{1}, \Pi_{5}\right]=-2 \Pi_{5}$, $\left[\Pi_{2}, \Pi_{5}\right]=2 \Pi_{4},\left[\Pi_{3}, \Pi_{4}\right]=-2 \Pi_{4}$, and $\left[\Pi_{3}, \Pi_{5}\right]=-2 \Pi_{5}$. Using that, we calculate Cartan-Killing form $K$ as follows:

$$
K=\left[\begin{array}{lllll}
6 & 0 & 6 & 0 & 0 \\
0 & 0 & 0 & 0 & 0 \\
6 & 0 & 8 & 0 & 0 \\
0 & 0 & 0 & 0 & 0 \\
0 & 0 & 0 & 0 & 0
\end{array}\right],
$$

where the determinant vanishes, and thus by Cartan criterion, it is not semisimple (see Proposition 4). Since a nilpotent Lie algebra has a Cartan-Killing form, that is, identically zero, we conclude, using the counter-reciprocal of the last claim, that the Lie algebra $\mathfrak{g}$ is not nilpotent.

We verify that the Lie algebra is solvable using the Cartan criteria to solvability (Proposition 5), and then we have a solvable non-nilpotent Lie algebra. The nilradical of the Lie algebra $\mathfrak{g}, M$ is generated by $\Pi_{2}, \Pi_{4}, \Pi_{5}$, and it is isomorphic to $\mathfrak{h}_{3}$, the Heisenberg Lie algebra, and so we have a solvable Lie algebra with three-dimensional nilradical. Let $m$ be the dimension of the nilradical $M$ of a solvable Lie algebra. In this case, in fifth-dimensional Lie algebra, we have that $3 \leq m \leq 5$.

Mubarakzyanov in [33] classified the 5-dimensional solvable non-nilpotent Lie algebras, in particular the solvable non-nilpotent Lie algebra with three-dimensional nilradical. Then, by Proposition 6 and consequently, we establish an isomorphism of Lie algebras with $\mathfrak{g}$ and the Lie algebra $\mathfrak{g}_{5,35}$. In summary, we have the next proposition.

Proposition 7. The 5-dimensional Lie algebra $\mathfrak{g}$ related to the symmetry group of equation (1) is a solvable non-nilpotent Lie algebra with three-dimensional nilradical; this nilradical is isomorphic to $\mathfrak{h}_{3}$, the Heisenberg Lie algebra. Besides that, Lie algebra is isomorphic with $\mathfrak{g}_{5,35}$ in the Mubarakzyanov's classification.

\section{Conclusion}

For a generalized Levinson-Smith equation (3), we obtained the optimal system's generating operators (see Proposition 2 ); using those operators, it was possible to characterize all invariant solutions as it is shown in Table 3; these invariant solutions do not appear in the literature known until today.

It has been shown in the variational symmetries for (3), as it was shown in (46) with its corresponding conservation laws (48) and all these were using Noether's theorem, but nontrivial conservation laws were also calculated using the Ibragimov's method as it is shown in (67) using the nonlinearly self-adjoint of equation (3) as mentioned in Proposition 3.

The results obtained in this study are new, and according to the phenomena that govern this equation, which reaches several fields of science, for instance, the nonlinear oscillators, it may be of significant importance for several researchers. Therefore, the goal initially proposed was achieved.

The Lie algebra associated to equation (3) is a solvable non-nilpotent Lie algebra with three-dimensional nilradical, and it is isomorphic with $\mathfrak{g}_{5,35}$ in the Mubarakzyanov's classification; therefore, the goal initially proposed was achieved.

For future works, equivalence group theory could be also considered to obtain preliminary classifications associated to a complete classification of (3).

\section{Data Availability}

The data used to support the findings of this study are included within the article.

\section{Conflicts of Interest}

The authors declare that they have no conflicts of interest.

\section{Acknowledgments}

The author Danilo A. García Hernández acknowledges the financial support from Coordenação de Aperfeiçoamento de Pessoal de Nível Superior, Brasil (CAPES), under the finance code 001. In the same way, the EAFIT University is thanked for all the financial supports (Scholarship for Master's Student). Moreover G. Loaiza and Y. Acevedo also thank the Project of MinScience "Sobre procesos de difusión y simplificación de información” (code 121671250122).

\section{References}

[1] S. Lie, "Theorie der transformationsgruppen," Mathematische Annalen, vol. 16, no. 4, pp. 441-528, 1880.

[2] E. Noether, "Invariante variationsprobleme. nachrichten der königlichen gessellschaft der wissenschaften," Mathematischphysikalishe klasse, vol. 2, pp. 235-257, 1918.

[3] N. H. Ibragimov, CRC Handbook of Lie Group Analysis of Differential Equations, CRC Press, Boca Raton, FL, USA, 1995.

[4] S. Dimas and D. Tsoubelis, "Sym: a new symmetry-finding package for mathematica," in Proceedings of the 10th International Conference in Modern Group Analysis, pp. 64-70, University of Cyprus Press, Larnaca, Cyprus, October 2004.

[5] Y. Ruo-Xia and L. Sen-Yue, "A Maple package to compute lie symmetry groups and symmetry reductions of (1+1)-dimensional nonlinear systems," Chinese Physics Letters, vol. 25, no. 6, pp. 1927-1930, 2008.

[6] G. Bluman and S. Kumei, "Symmetries and differential equations," in Applied Mathematical SciencesSpringer, New York, NY, USA, 1989.

[7] L. Ovsyannikov, Group Analysis of Differential Equation, Academic Press, New York, NY, USA, 1982.

[8] B. J. Cantwell, "Introduction to symmetry analysis," in Cambridge Texts in Applied MathematicsCambridge University Press, Cambridge, UK, 2002. 
[9] H. Stephani, Differential Equations: Their Solution Using Symmetries, Cambridge University Press, Cambridge, UK, 1989.

[10] P. G. L. Leach and A. Paliathanasis, "Symmetry analysis for a fourth-order noise-reduction partial differential equation," Quaestiones Mathematicae, vol. 0, no. 0, pp. 1-12, 2020.

[11] A. Paliathanasis and P. G. L. Leach, "Symmetries and singularities of the szekeres system," Physics Letters A, vol. 381, no. 15, pp. 1277-1280, 2017.

[12] A. Ghose-Choudhury, P. Guha, A. Paliathanasis, and P. G. L. Leach, "Noetherian symmetries of noncentral forces with drag term," International Journal of Geometric Methods in Modern Physics, vol. 14, no. 2, Article ID 1750018, 2017.

[13] W. Hu, Z. Wang, Y. Zhao, and Z. Deng, "Symmetry breaking of infinite-dimensional dynamic system," Applied Mathematics Letters, vol. 103, Article ID 106207, 2020.

[14] E. Alimirzaluo, M. Nadjafikhah, and J. Manafian, "Some New Exact Solutions of \$(3+1)\$-dimensional Burgers System via Lie Symmetry Analysis," in Advances in Difference EquationsSpringer, Berlin, Germany, 2021.

[15] A. Paliathanasis, "Lie symmetry analysis and one-dimensional optimal system for the generalized $2+1$ kadomtsev-petviashvili equation," Physica Scripta, vol. 95, no. 5, Article ID 055223, 2020.

[16] H. Lu and Y. Zhang, "Lie symmetry analysis, exact solutions, conservation laws and bäcklund transformations of the gibbons-tsarev equation," Symmetry, vol. 12, no. 8, p. 1378, 2020.

[17] S.-F. Tian, "Lie symmetry analysis, conservation laws and solitary wave solutions to a fourth-order nonlinear generalized boussinesq water wave equation," Applied Mathematics Letters, vol. 100, Article ID 106056, 2020.

[18] M. R. Ali and R. Sadat, "Lie symmetry analysis, new group invariant for the $(3+1)$-dimensional and variable coefficients for liquids with gas bubbles models," Chinese Journal of Physics, vol. 71, pp. 539-547, 2021.

[19] E. Kamke, Differential gleichungen: Losungs methoden und Losungen, Gewohnliche Differential gleichungen, B. G. Teubner, Leipzig, Germany, 1977.

[20] A. Liènard, "Etude des oscillations entretènues," Revue gènèrale de l'èlectricitè, vol. 23, pp. 901-912, 1928.

[21] N. Levinson and O. K. Smith, "A general equation for relaxation oscillations," Duke Mathematical Journal, vol. 9, no. 2, pp. 382-403, 1942.

[22] P. J. Olver, Applications of Lie Groups to Differential Equations, Springer-Verlag, Berlin, Germany, 1986.

[23] P. Hydon and D. Crighton, "Symmetry methods for differential equations: a beginner's guide," in Cambridge Texts in Applied MathematicsCambridge University Press, Cambridge, UK, 2000.

[24] Z. Hussain, Optimal System of Subalgebras and Invariant Solutions for the Black-Scholes Equation, Blekinge Institute of Technology, Karlskrona, Sweden, 2009.

[25] G. Zewdie, Lie Simmetries of Junction Conditions for Radianting Stars, University of KwaZulu-Natal, Durban, South Africa, 2011.

[26] E. Noether, "Invariante variationsprobleme, königlich gesellschaft der Wissenschaften," Göttingen Nachrichten Mathematik-Physik Klasse, vol. 2, pp. 235-267, 1918.

[27] M. C. Nuccci and P. G. L. Leach, "An old method of Jacobi to find Lagrangians," Journal of Nonlinear Mathematical Physics, vol. 16, no. 4, pp. 431-441, 2009.

[28] I. M. Gelfand and S. V. Fomin, Calculus of Variations, Dover Publications, Garden City, NY, USA, 2000.
[29] M. L. Gandarias, "Weak self-adjoint differential equations," Journal of Physics A: Mathematical and Theoretical, vol. 44, Article ID 262001, 2011.

[30] N. H. Ibragimov, "A new conservation theorem," Journal of Mathematical Analysis and Applications, vol. 333, pp. 311328, 2007.

[31] N. H. Ibragimov, "Nonlinear self-adjointness in constructing conservation laws," Archives of ALGA, vol. 7/8, pp. 1-90, 2011.

[32] J. E. Humphreys, Introduction to Lie Algebras and Representation Theory, Springer, Berlin, Germany, 2012.

[33] G. M. Mubarakzyanov, "Classification of real structures of lie algebras of fifth order," Izvestiya Vysshikh Uchebnykh Zavedenii, vol. 3, no. 1, pp. 114-123, 1963. 